\title{
Gradhiva
}

\section{Morgan Jouvenet, Rap, techno, électro, le musicien entre travail artistique et critique sociale, préface de Pierre-Michel Menger}

Paris, Éditions de la Maison des sciences de l'homme («Ethnologie de la France »), 2006, XX-290 p.

\section{Denis-Constant Martin}

\section{(2) OpenEdition}

\section{Journals}

Édition électronique

URL : http://journals.openedition.org/gradhiva/973

DOI : $10.4000 /$ gradhiva.973

ISSN : 1760-849X

\section{Éditeur}

Musée du quai Branly Jacques Chirac

Édition imprimée

Date de publication : 15 novembre 2007

Pagination : 126-127

ISBN : 978-2-915133-55-4

ISSN : 0764-8928

\section{Référence électronique}

Denis-Constant Martin, « Morgan Jouvenet, Rap, techno, électro, le musicien entre travail artistique et critique sociale, préface de Pierre-Michel Menger », Gradhiva [En ligne], 6 | 2007, mis en ligne le 10 décembre 2008, consulté le 21 septembre 2020. URL : http://journals.openedition.org/gradhiva/973 ; DOI : https://doi.org/10.4000/gradhiva.973

Ce document a été généré automatiquement le 21 septembre 2020

(c) musée du quai Branly 


\section{Morgan Jouvenet, Rap, techno, électro, le musicien entre travail artistique et critique sociale, préface de Pierre-Michel Menger}

Paris, Éditions de la Maison des sciences de l'homme («Ethnologie de la France »), 2006, XX-290 p.

Denis-Constant Martin

\section{RÉFÉRENCE}

Morgan Jouvenet, Rap, techno, électro, le musicien entre travail artistique et critique sociale. Paris, Éditions de la Maison des sciences de l'homme («Ethnologie de la France »), 2006, XX-290 p.

1 L'université française a mis (trop) longtemps à accepter la légitimité des recherches portant sur des musiques diversement qualifiées de «populaires, de masse, actuelles, amplifiées "; on ne peut donc que se réjouir de voir publier des thèses qui leur ont été consacrées et devraient permettre d'insérer la réflexion française dans les débats suscités, depuis plusieurs décennies, par ces musiques en Europe et en Amérique. C'est sur cet arrière-plan qu'il convient d'apprécier l'ouvrage de Morgan Jouvenet, issu d'une thèse soutenue en 2003. L'auteur a choisi d'aborder le rap et les musiques électroniques sous l'angle de la production, en liant fort justement production artistique (des genres, de l'œuvre) et production industrielle et commerciale. Il commence donc par tenter de définir les genres qui vont être étudiés et retrace leurs origines, à l'étranger (surtout aux États-Unis) et en France. Il insiste ensuite sur ce qu'ils représentent de plus novateur: une attitude nouvelle face au matériau musical, des manières de faire (au sens de fabriquer) plus que des arts du penser la musique (auxquels se rattacherait la composition au sens traditionnel). Ces approches doivent beaucoup aux changements 
techniques et culturels qui se sont produits depuis la fin de la seconde guerre mondiale : développement de cultures cinématographiques et télévisuelles, invention de nouvelles lutheries utilisant les ressources de l'électronique et de l'informatique. Ces innovations, et les pratiques qu'elles ont stimulées, ont donné à de nouvelles générations de "musiquants» (artisans de la musique : "musiciens» au sens étroit, mais aussi producteurs, diffuseurs et "amateurs" de plus en plus difficilement distingués des "musiciens" professionnels) des moyens considérables pour créer, souvent au départ sur le mode du bricolage, de nouvelles formes musicales. Pour eux, l'œuvre a perdu son caractère intangible, quasiment sacré ; la musique est un « objet manipulable "; le son est ressenti comme " un espace à habiter »; composer revient à «transformer et [à] sélectionner »; le résultat de l'activité musicale demeure ouvert, recyclable (p. 67-68).

2 En pratique, la production musicale est devenue nécessairement collective, impliquant diverses catégories d'acteurs, et la performance elle-même se veut, et entend se montrer, interactive. Ces ruptures par rapport aux conventions qui dominaient non seulement la musique « classique » mais aussi le jazz ou la chanson sous-tendent, disent rappeurs et électronistes, des visions insoumises de la "société de consommation »: si les musiques électroniques favorisent une "mise à distance de la réalité » (p. 92), les rappeurs souhaitent provoquer une "prise de conscience " grâce à des textes qui portent un "dévoilement critique» (p. 101). Tous se réclament d'une éthique de l'authenticité : les rapports noués entre différents acteurs de la production musicale doivent être transparents pour les consommateurs; les relations entre artistes sont affichées sur scène ou mises en textes; les médias les publient. Pourtant, ces musiques n'échappent pas à la commercialisation et aux déformations de la médiatisation. Si l'idéal de la firme de production discographique spécialisée est l'indépendance, avec des modes de fonctionnement internes souples et conviviaux, la réalité est celle d'une répartition des tâches entre majors et « indies » indépendants, prenant la forme d'un « oligopole à franges » (p. 168) qui formate des artistes dont un certain « sérieux » est exigé s'ils veulent être efficacement pris en charge, et qui pèse sur les choix et les évaluations des médias. Dans le rap et les musiques électroniques se retrouve donc une tension (qui trame d'autres genres : chanson, rock, jazz, et même aujourd'hui musique "classique») entre conformisme et création, puisque l'artiste doit être à la fois " original et prévisible ». De ce fait, l'« authenticité », bien que " principe organisant les mondes du rap et de l'électronique » (p. 266), se révèle être un mythe, partie intégrante d'un discours idéologique largement répandu aujourd'hui dans la publicité, dans la politique et accompagnant également d'autres genres ou catégories musicaux (la World Music, par exemple).

3 Le lecteur peut donc s'étonner que la conclusion de ce livre, intitulée précisément "Pour ne pas conclure : 1'“authenticité" en question », n'interroge pas davantage les prétentions à l'authenticité, à la transparence, à la pureté qui apparaissent dans les discours des "musiquants ", et ne remarque pas que ce thème doit être saisi comme un aspect d'un phénomène bien plus vaste qui affecte nombre de sociétés contemporaines dites développées. Ces «absences » signalent, plus largement, des problèmes de méthode qu'une sociologie du travail artistique ne peut éluder. Le premier d'entre eux porte sur la place de l'analyse musicale. D'entrée de jeu, l'auteur, qui a posé le genre musical comme un "objet fuyant", affirme, sans justifier son jugement, que "la tentation musicologique se révèle stérile» (p. 9). C'est faire fi d'un grand nombre de 
recherches en anthropologie ou en sociologie de la musique, publiées notamment en anglais, et refuser de considérer les leçons que la sociologie de la musique peut tirer de l'ethnomusicologie ; l'auteur nous démontre d'ailleurs qu'il aurait mieux fait de ne pas si fort résister à cette tentation en enchaînant immédiatement, à propos du rap, que «le phrasé saccadé du chanteur suffit à identifier le genre » (p. 9-10), ce qu'il précise à peine quelques pages plus tard : « $[\mathrm{MBO}]$ un mode très particulier de diction, par lequel le chanteur débite rapidement un texte, de manière saccadée et sans grande variation de ton.» (p. 17) Suivant cette définition, le rap serait une pratique très largement répandue dans les chansons françaises ou américaines et remonterait au moins au fameux « Je n'suis pas bien portant» de Gaston Ouvrard... Travailler sur les musiques populaires aujourd'hui commercialisées demande effectivement de définir les genres que l'on y distingue. Ces genres font l'objet d'étiquettes communément utilisées par musiciens, producteurs, journalistes, disquaires et amateurs; celles-ci doivent évidemment être prises en considération; elles doivent aussi être analysées à la lumière d'une catégorisation musicale dont le but est de mettre en évidence (ou de montrer qu'ils ne sont pas présents) des traits caractérisant les genres envisagés. C'est à partir du rapport entre étiquettes commerciales et catégories musicales que pourront être analysés les sens (sens commercial, sens symbolique notamment) des étiquettes courantes. L'analyse musicale n'est pas indispensable à toute sociologie de la musique mais, s'il faut définir des genres musicaux, on ne peut en faire l'économie qu'au prix de l'imprécision.

Le corollaire du refus de la «tentation musicologique » est, chez Morgan Jouvenet, le travail à partir des "descriptions indigènes" (p. 9). Second problème: si aucune sociologie ne peut négliger le discours des acteurs, toutes (y compris la sociologie de la musique) doivent l'analyser et non le traiter comme source d'information. Ce discours est un récit, imprégné de représentations, porteur d'aspirations, visant à projeter une image du locuteur et de ce qu'il fait. Il est d'autant plus construit qu'il est tenu devant un journaliste et remis en forme par ce dernier dans l'entretien finalement publié (Morgan Jouvenet signale, p. 244, ce qu'implique «savoir parler aux interviews »). Lorsqu'il se veut «critique » ou insoumis (p. 86-87), lorsqu'il prétend façonner la musique selon des esthé-

tiques «sociologistes» (p. 80), ce discours doit être confronté aux pratiques, aux productions, voire à la vie des artistes. C'est cette confrontation qui fait ici défaut car on ne trouve dans cet ouvrage ni analyse symbolique de la musique, ni analyse du contenu de textes, ni analyse structurelle de performances, ni biographies de rappeurs ou d'électronistes qui permettraient de reconstituer les parcours sociaux sur l'arrièreplan desquels leurs déclarations sont formulées. De même, la présentation des systèmes de production ne contient pas d'analyse organisationnelle des « indépendants » ou des majors, et les proclamations identitaires lancées par les rappeurs ne sont pas évaluées à l'aune des réactions qu'elles provoquent chez leurs auditeurs. Les réalités qui sont au cœur de ce travail sont abordées par le biais de discours, sans que ce biais soit lui-même l'objet d'une approche critique d'autant plus nécessaire que sont fréquemment utilisés des entretiens publiés dans la presse.

Il faut saluer le livre de Morgan Jouvenet comme un travail pionnier, une des premières tentatives pour aborder sérieusement en français, dans un cadre universitaire, des musiques trop souvent négligées, voire méprisées, par des générations anciennes de musicologues ou d'ethnomusicologues. Sa lecture fournit une quantité appréciable d'informations sur le traitement par les industries de la musique de genres dont la 
popularité assure qu'ils représentent indiscutablement des phénomènes sociaux, sur la manière dont y œuvrent différentes catégories de créateurs, sur les façons dont ils cherchent à y construire leur image. L'éclairage porté sur le rap et les musiques électroniques en termes de manières de faire, s'il n'est pas entièrement nouveau, n'en est pas moins fertile. Et, imparfait comme tout travail pionnier, il ouvre des pistes de réflexion, notamment méthodologiques, quant à ce que peut et doit être une sociologie des musiques populaires commerciales. Dans l'ensemble, ses lacunes invitent à conjuguer la luxuriance théorique et l'imagination interprétative à un empirisme plus rigoureux: analyse musicale, lorsqu'elle s'avère nécessaire; investigations dans les organisations; recueil et analyse de récits de vie des acteurs; enquête chez les auditeurs. Bref, à aborder la musique comme un objet sociologique ordinaire mais à partir de ses spécificités.

\section{AUTEURS}

\section{DENIS-CONSTANT MARTIN}

denisconstant@club-internet.fr 